\title{
EUV mask reflectivity measurements with micron-scale spatial resolution
}

\author{
Kenneth A. Goldberg ${ }^{1}$, Senajith B. Rekawa' ${ }^{1}$ Charles D. Kemp ${ }^{1}$, Anton Barty ${ }^{2}$, \\ Erik Anderson', Patrick Kearney ${ }^{3}$, Hakseung $\mathrm{Han}^{3}$ \\ 'Center for X-Ray Optics, Lawrence Berkeley National Laboratory, Berkeley, CA 94720 \\ ${ }^{2}$ Lawrence Livermore National Laboratory, P.O. Box 808, Livermore, CA 94550 \\ ${ }^{3}$ SEMATECH, 255 Fuller Road, Suite 309, Albany, NY 12203
}

\begin{abstract}
The effort to produce defect-free mask blanks for EUV lithography relies on increasing the detection sensitivity of advanced mask inspection tools, operating at several wavelengths. We describe the unique measurement capabilities of a prototype actinic (EUV wavelength) microscope that is capable of detecting small defects and reflectivity changes that occur on the scale of microns to nanometers. The defects present in EUV masks can appear in many well-known forms: as particles that cause amplitude or phase variations in the reflected field; as surface contamination that reduces reflectivity and contrast; and as damage from inspection and use that reduces the reflectivity of the multilayer coating. This paper presents an overview of several topics where scanning actinic inspection makes a unique contribution to EUVL research. We describe the role of actinic scanning inspection in defect repair studies, observations of laser damage, actinic inspection following scanning electron microscopy, and the detection of both native and programmed defects.
\end{abstract}

Keywords: extreme ultraviolet lithography, EUV, mask inspection, reticle, defect repair, actinic inspection, bright-field, dark-field.

\section{INTRODUCTION}

The optical coatings and materials used in EUV masks exhibit strongly wavelength-dependent effects, including resonant reflectivity and thickness-dependent phase-shifts that sensitively affect aerial images that sensitively affect aerial images. Research on the development of EUV-mask inspection tools benefits by careful cross-correlation of measured results with EUV photolithographic printing ${ }^{1}$ and actinic (EUV-wavelength) mask inspection.

While the availability of high speed, commercial, actinic (EUV-wavelength) mask inspection systems is still several years away, existing and new tools that use ultraviolet light detection methods continue to make important advances in detection sensitivity ${ }^{2,3}$. Yet for ultraviolet inspection, with source wavelengths that are nearly twenty times longer than EUV light wavelengths, the measurement response to different types of defects is a subject of active research. Particles on the substrate (below the multilayer coating) or the top surface are only two of the many classes of defects that can affect printed features. There is also interest in damage caused by inspection and repair, which can occur in the form of small-scale reflectivity variations, and changes that only observable at-wavelength. While it is clear that micron-scale reflectivity changes of sufficient magnitude can affect the aerial image, little attention has been paid to this topic in part because no data has been available.

\section{AIT SYSTEM DESIGN}

The Sematech Berkeley Actinic Inspection Tool (AIT) is a dual-mode EUV-wavelength microscope, with scanningbeam and (zoneplate) imaging capabilities. The AIT is located at Lawrence Berkeley National Laboratory's Advanced Light Source (ALS) synchrotron, and operates on a bending magnet beamline that provides stable, tunable, narrow-band illumination (up to $\triangle E / E \sim 1 / 1500$ ) centered around 13.4-nm wavelength. 

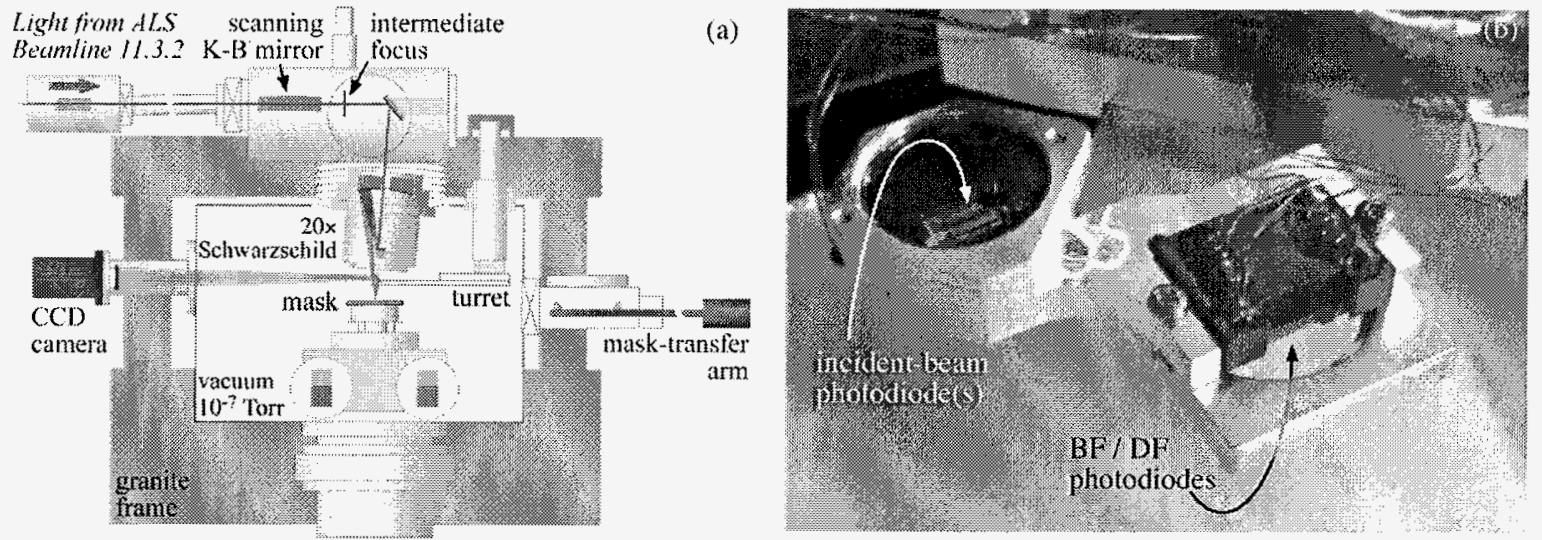

Fig. 1 The AIT Scanning Mode Configuration. (a) Bending-magnet beamline 11.3.2 at the ALS provides tunable, monochromatic EUV light to the AIT. A K-B mirror pair brings the beam to an intermediate focal point above the main chamber. A $20 \times$ Schwarzschild objective serves as an illuminator, re-imaging the K-B focus to the mask with a divergence angle up to $0.0625 \mathrm{NA}$. Essential optical elements for the microscope rotate and translate on a 4-axis turret arm into the $2-\mathrm{cm}$ gap between the mask and the Schwarzschild objective. The CCD camera attached to the chamber is used in imaging mode. In scanning mode a photodiode is held on the end of the turret arm. The system operates at a base pressure of $6 \times 10^{-7}$ Torr backfilled with oxygen to $6 \times 10^{-5}$ Torr during operation.

(b) Identical $3 \times 1$ element photodiode arrays are mounted to intercept either the incident beam or the reflected beam. Each diode element has $1 \times 1 \mathrm{~mm}^{2}$ area. The diodes are positioned so that one element measures the specular beam, while an adjacent element sits just outside of the specular beam's solid angle. Phosphor paint covers many edges of the device to visually detect the beam location through the chamber view-ports.

(c) A Schematic shows that the $6^{\circ}$ incident angle of the central ray leaves only a small clearance for the photodiode to intercept the beam.

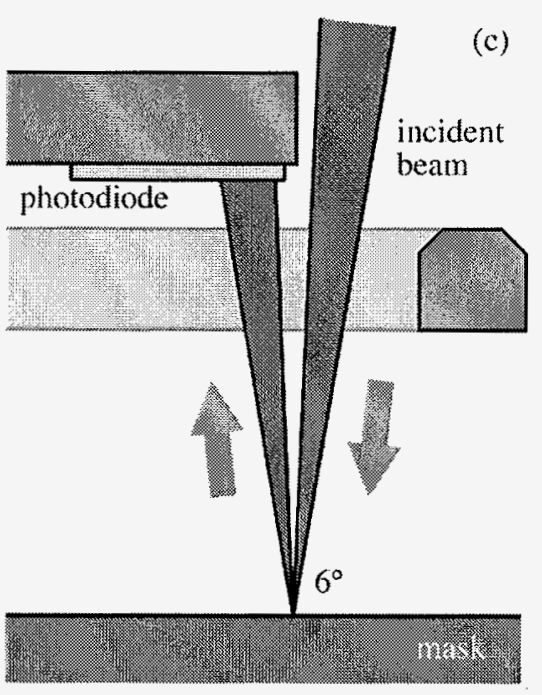

In scanning beam mode, narrow-band EUV light is focused onto the mask surface with $6^{\circ}$ incidence angle, and a spot size from $1-5 \mu \mathrm{m}$ in diameter, as shown in Figs. la and 1c. As the mask is scanned laterally, the stationary focused beam moves relative to the mask surface, and the reflected light is recorded by a $3 \times 1$ array of $1 \mathrm{~mm}^{2}$ square photodiodes (Fig. 1b). These photodiodes are significantly larger than the beam size in that plane. Because of the small clearance, the downward-facing diode substrate was cut to bring the elements close to the edge of the holder.

The AIT can record the signal from two of the downward-facing diodes simultaneously. One diode is positioned to detect the specular beam; it measures the bright-field local reflectivity changes of the mask surface. ${ }^{2,4}$ An adjacent diode is positioned just outside of the specular beam where it captures dark-field scattering and light re-directed outside of the specular beam's solid angle, in one direction. The bright-field measurements are sensitive to local reflectivity changes with uncertainties as small as $0.1 \%$, averaged over the beam diameter. Dark-field measurement sensitivity depends on the detection solid angle (adjustable by positioning the diode) and the sizes of the features being studied. Dark-field detection is limited by electronic noise in the detection and amplification of the small $(1-100 \mathrm{pA})$ signal.

The mask position under the stationary beam is controlled by a rotation and translation stage, $x \theta z$. Longitudinal motion ( $z$, through focus) is straightforward, but lateral motion ( $x y$ ) must be performed as a combination of rotation and translation. For this reason, measurement at arbitrary mask locations requires an azimuthally rotated mask orientation dictated by the system geometry.

Since the beamline focus is re-imaged onto the mask by the Schwarzschild illumination optics, the size of the beam footprint on the mask is determined by the aperture size used in the intermediate focus of the beamline. With $20 \times$ demagnification from the Schwarzschild illuminator, the 100,50 , and $20 \mu \mathrm{m}$ circular aperture sizes, create 5, 2.5, and 
$1.0 \mu \mathrm{m}$ beam footprints on the mask respectively. Since the beam size is slightly larger than the largest pinhole size, the apertures also restrict the transmitted flux in proportion to their area. Therefore, while the flux density on the mask remains roughly constant for all beam sizes, the experiment faces a trade-off between spatial resolution and the available light power, which largely determines the signal-to-noise ratio.

The upward-facing photodiode was added in 2007 as a means to periodically measure, and thereby calibrate, the incident flux level. At this time, adequate calibration has not been achieved, and the scanning measurements are limited to relative, rather than absolute, reflectivity changes on the mask surface.

The scanning speed depends on many factors, but it is primarily limited by the current amplifier bandwidth. Detected signal levels in bright-field scanning are as high as $10 \mathrm{nA}$, and are typically in the 1-5-nA range. Darkfield signal strengths may be 100 times smaller. To achieve high signal-to-noise ratios it is necessary that the mask be scanned slowly beneath the beam. Normally the linear speed of the mask is limited to several microns per second. The time required to scan an area depends on the scanning path, including the scan-line density, which is continuously adjusted: coarse to identify regions of interest, and fine to match the spatial scale of the features being studied.

Accurate data processing in scanning mode is complex, and is briefly discussed in Section 3.1. The primary challenge for scanning measurement accuracy is the intensity-level stability of the illumination over time. Since the beamline light is focused onto a pinhole, the delivered flux is sensitive to the beamline's alignment stability, and any drift or vibration in the upstream alignment can change the transmitted flux. The beamline overfills the pinhole by a factor of $2-5$ horizontally and 10-50 vertically, and the angular demagnification by the K-B optics also reduces misalignment sensitivity. Furthermore, changes in the synchrotron's storage ring current directly affect the flux delivered to the sample.

In practice, we observe slow, unpredictable fluctuations in the flux level, up to several percent, that can decrease (and restore) the recorded photocurrent-changes typically occur on a time-scale of minutes. Aftempts to use photocurrent from beamline mirrors as a normalization signal have been complicated by the nonlinearity of the photocurrent response. Therefore, signal normalization is a critical issue in achieving accurate measurements.

During scanning, the beam follows a serpentine (back-and-forth) path across the mask surface. Because the signal is continuously acquired and recorded during the scan, the sampling density is high along the scan lines, and coarse in the perpendicular direction. Considering the presence of electronic noise and the unpredictable temporal changes in the beam intensity, surface reconstruction from the scanning data presents its own set of complications.

When open areas of a mask blank are scanned, assumptions about the uniformity of the coating allow us to perform normalization from the data signal itself. Isolated defects stand out from the background signal level. However, when dense patterned regions are scanned, normalization becomes significantly more difficult; fluctuations in the illuminating intensity are difficult to separate from the pattern itself. In general, pattern and feature edges (i.e. abrupt reflectivity changes) are the most reliable measurements because instantaneous signal changes can be attributed to the sample.

Regarding dark-field detection, we have found that it is difficult to predict the detector position that optimizes the darkfield response for a given measurement. As the dark-field detector element is placed farther away from the specular beam, the signal level from background scattering, and from the measured features, drops quickly. On the other hand, if the detector is placed too close to the specular beam, it ceases to serve as a dark-field detector and acts more like a knifeedge. (We acknowledge that this knife-edge configuration may be interesting for its high sensitivity, but it does provides neither reliable dark-field measurements of small scattering objects nor bright-field reflectivity measurements.) In practice, we determine the 'compromise' detector position empirically by performing multiple scans and identifying the location where the response to a given set of defects is highest. This strategy can be adjusted to suit specific measurements, but repeatability in the dark-field detector position is an alignment challenge for the AIT.

\section{SCANNING-MODE MEASUREMENTS}

This section surveys the different kinds of measurements that have been performed in the AIT's scanning mode. A few of these results have been described previously in greater detail, but with a different emphasis. Here the goal is to show a variety measurement capabilities and interesting research problems that can be addressed with the AIT, more than to elucidate the detailed results. 


\subsection{Laser damage and the issue of scanning-data normalization}

High-powered ultraviolet lasers are used in commercial mask blank inspection tools, and the potential for mask damage is an issue warranting special attention from actinic inspection tools. Laser power levels can be increased to improve the signal to noise ratio in the detection of light scattered from the smallest visible defects. However, the potential for mask damage is significant since the damage caused by inspection may not be apparent or observable at the inspection wavelengths. ${ }^{5}$

We have conducted actinic scanning to measure EUV reflectivity changes induced by exposure to ultraviolet laser wavelengths. These tests are essential in setting the power thresholds below which damage is either imperceptible or tolerable. In an ultraviolet mask blank inspection system, light is highly concentrated, so the damage areas may have feature sizes as small as $5-100-\mu \mathrm{m}$ in size. Reflectivity changes on this small size scale may not be easy to detect in conventional reflectometers, which use larger beam footprints (50-500- $\mu \mathrm{m}$ wide, or more).
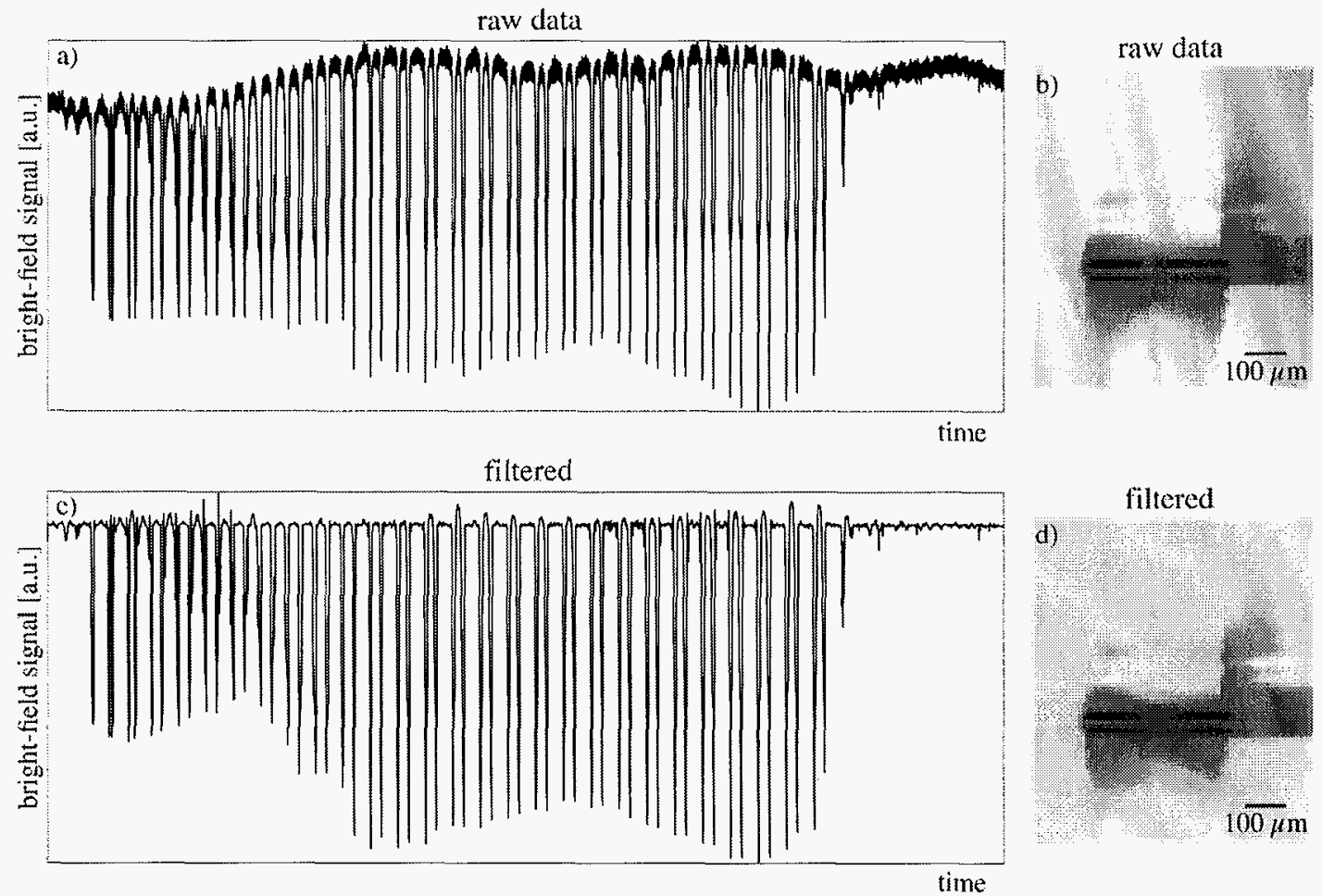

Fig. 2. Large area scans of a region showing high-powered UV laser damage, raw and filtered -data reconstructions. Without filtering (a, b), fluctuations in the illumination flux level can appear as large-scale changes in the apparent reflectivity that follow the direction of the scanning path, slightly inclined from vertical. Intensity-level filtering (c, $d$ ) is most effective in regions surrounded by open mask areas. In this example, the largest reflectivity change occurs in the dark regions, where the reflectivity falls to $31 \%$ of the background level.

Figure 2 shows scanning data from a $700 \times 790-\mu \mathrm{m}$ mask region where laser damage reduces the EUV reflectivity. Figure 2a shows the (raw) current measured by the photo-diode during the scan, and a reconstruction of the local reflectivity variation based on the scan data and the measured encoder positions. The scan shown here is relatively coarse, with $10-\mu \mathrm{m}$ scan steps. Light areas are regions with full, reflectivity magnitude. The reflectivity falls as low as $31 \%$ of the background level in the regions rendered as black.

Figure 2a also shows an example of the intensity fluctuations associated with the beamline alignment instability. The total data collection time shown is 105 seconds. The intensity data in Fig. 2a fluctuated by $4.7 \%$ of the average background level during the scan. As shown here, the serpentine scan path follows a nearly vertical trajectory through the region and moves slowly from right to left. Without filtering, this fluctuation creates a light and dark banding (Fig. 2b) effect that mimics large-spatial-scale reflectivity variations. 
Figure $2 \mathrm{c}$ is a filtered version of the raw data, used to reconstruct Fig $2 \mathrm{~d}$. With each pass through the laser-damaged region, the reflectivity drops and returns to the background level, so the relative magnitude in these abrupt spatial changes can be measured accurately. (This is what gives rise to the comb pattern in the plot).

Figures $2 c$ and $d$ show a filtered version of the same data and a reconstruction from it. Two filters are applied to create the signal shown in $2 \mathrm{c}$. First, the data is median-filtered to remove spurious spikes and noise. The temporal width of the median filter is always much less than the filtering time-scale of the current amplifier to ensure that real data is not compromised. The second filter addresses the intensity fluctuations by extracting the peak levels within relatively large time bands and using them for normalization. This filter uses the assumptions that (a) the background levels outside of the damage region is homogenous, and (b) it can be used for normalization. The filter performs best when at least some portion of each scan line includes the background region.

\subsection{SEM Inspection Damage}

It is well known that scanning electron microscope (SEM) inspection can induce carbon deposition onto surfaces. ${ }^{6}$ During SEM inspection, regions studied at high resolution (and thus high electron flux density) often appear noticeably darker in SEM micrographs. This staining is typically caused by the deposition of a thin layer of carbon caused by the interaction of primary or secondary electrons with residual hydrocarbons in the sample chamber. What is not well known is how that damage affects EUV reflectivity.

A simple test was conducted in which a patterned EUV reticle was inspected in a commercial SEM, creating easily identifiable stained regions, and then an actinic scan was performed on the same region. Several high-resolution images were collected in regions that included an $1-\mu \mathrm{m}$ square contact, and the end of a $2.5-\mu \mathrm{m}$ bright line (Fig. 3a). Actinic scans were performed in bright-field mode only, using a $2.5 \mu \mathrm{m}$ beam diameter. Only limited time was available for this test, and repeated measurements of the region were not conducted. The elliptical beam footprint evident in the appearance of the scanned contacts may be caused by astigmatism in the illumination system.

In the single actinic scan acquired for this measurement (Fig. 3b), no evidence of the prior SEM inspection could be detected within several percent measurement uncertainty. Neither the contact nor the line-end appeared to be detectably different from similar adjacent features.

One reason for the negligible difference may be the relatively high transparency of carbon to EUV light. The attenuation length in carbon for 13.4-nm wavelength light is approximately $158 \mathrm{~nm}^{7}$. Thus the absorption upon reflection through a 1 -nm carbon film is just $1.26 \%$.

A second reason may be the difficulty of achieving accurate signal normalization within patterned measurement regions. Had the tests been conducted within strictly open mask areas, small changes may have been more easily detected; follow-up tests should be conducted to confirm this supposition.
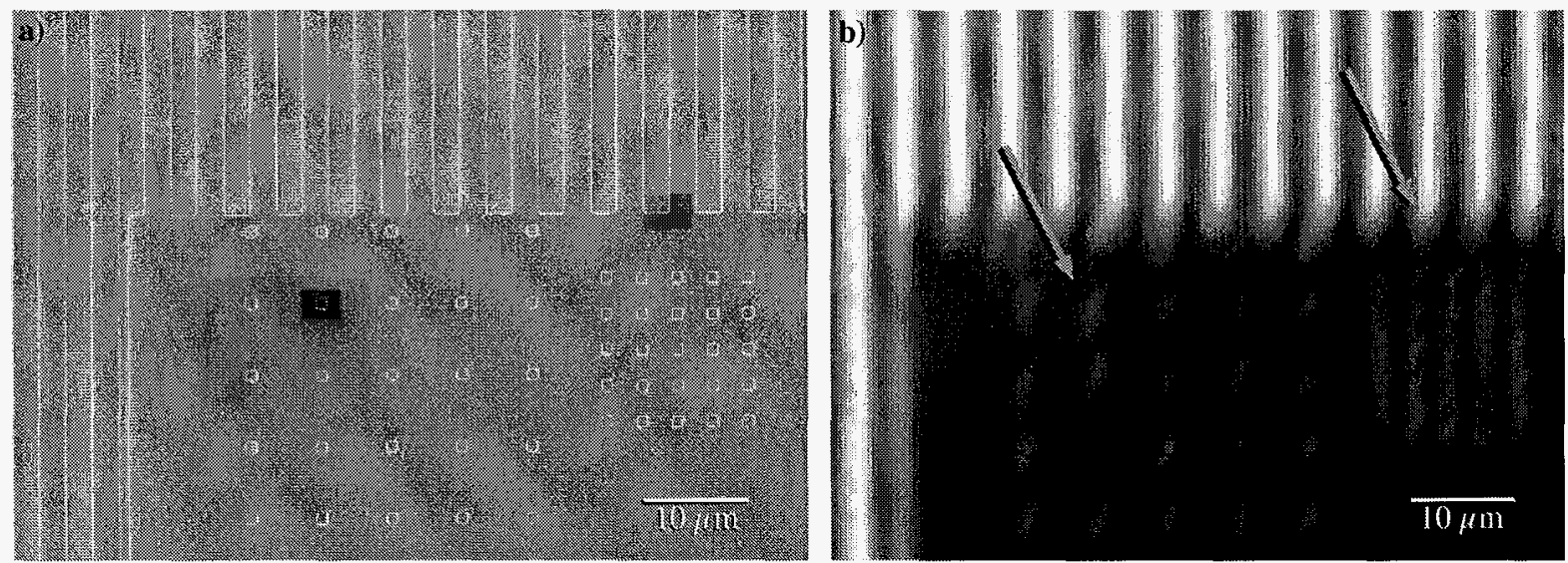

Fig. 3. (a) SEM and (b) actinic bright-field scan of the same region of a patterned EUV mask. High-resolution SEM inspection was performed to induce intentional carbon contamination staining on the surface. Subsequent actinic bright-field inspection did not detect a decrease in the EUV reflectivity, within the measurement uncertainty. The contacts are $1-\mu \mathrm{m}$ square, and the lines are $2.5-\mu \mathrm{m}$ wide. The EUV beam diameter was $2.5 \mu \mathrm{m}$, and some astigmatism is apparent. 
While this single test was limited in scope, the absence of significant EUV reflectivity changes, despite the observable carbon-deposition staining in the SEM, shows that high-resolution SEM inspection, when performed quickly, may not be a severe threat to the quality of EUV masks.

\subsection{Open-field mask blank repair strategies}

As reported in $2007^{8}$ the AIT has been used to probe the EUV response of prototype, open-field, mask blank defect repair strategies. Working in collaboration with researchers from Carl Zeiss and AMD, we found that the EUV bright-field (reflectivity) and dark-field (scattering) response to the repair sites could be markedly different, and uncorrelated. Such information has significant bearing on the effectiveness of proposed actinic inspection tools that rely solely on either bright-field or dark-field measurements.

In the experiment, a multilayer-coated EUV mask blank was prepared containing an array of fourteen defects and repair sites. The mask was coated by the SEMATECH North Mask Blank Development Center (MBDC). The repair involved an e-beam-activated chemically induced etching process that can be controllably localized at the size of the defect. The process was developed by Carl Zeiss SMS and performed on a mask repair tool prototype. The goal was to create repairs with sidewall slopes below $4^{\circ}$, which has been calculated as the largest acceptable angle that does not create phase shift errors 9 Repairs were performed in an open loop procedure (not using feedback) without tight control of surface roughness. (It should be noted that an adequate mask-repair recipe was not identified in these first measurements. ${ }^{5}$ )

The repair sites themselves were circular marks etched into the mask and treated in different ways to distinguish effects of the etching into the multilayer from the effects of a protection layer. The etch and repair site diameters varied from 2 to $6 \mu \mathrm{m}$.

Measurements of two different sites are shown in Fig. 4. The site on the left is an etched pit, $4 \mu \mathrm{m}$ diameter, with $2-4^{\circ}$ sidewall angles, and no repair. The site on the right, with a $5-\mu \mathrm{m}$ pit diameter, had similar etching and was covered with a 6- $\mu \mathrm{m}$-diameter $\mathrm{SiO}_{2}$ protection layer, approximately 5-nm thick. Both sites were easily detected in the Lasertec M1350, which operates at 488-nm wavelength.

To improve the signal-to-noise ratio of the actinic dark-field detection, a 5- $\mu \mathrm{m}$ diameter was used in these measurements. The bright-field signal gains spatial discrimination and sensitivity when a smaller beam is used; thus for brightfield measurement we performed separate scans with a $1-\mu \mathrm{m}$-diameter beam.

The key statistics from the actinic scans are the bright-field peak reflectivity loss, labeled "BF $\Delta R$," and the dark-field signal strength (signal-to-noise ratio), labeled "DF SNR" in Fig. 4. For the bright-field data, the relative reflectivity is normalized to the clear mirror areas adjacent to the defect sites.

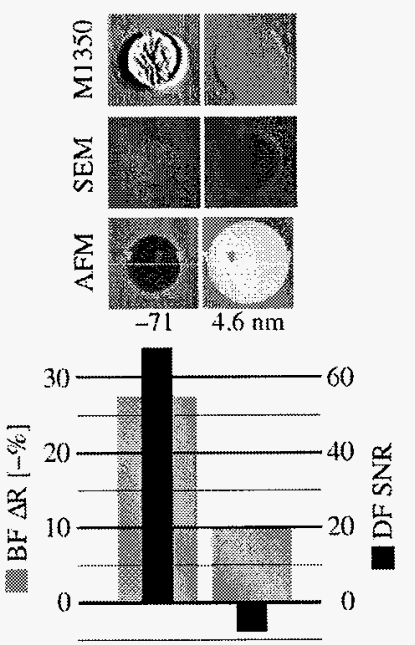

Fig. 4. Two sites on a reticle-repair test mask investigated with actinic and nonactinic inspection. "M1350" images were recorded in the Lasertec M1350's defectreview mode. SEM and AFM measurements are also shown. AFM-measured peak surface displacements are shown in $\mathrm{nm}$. Each image is approximately $6-\mu \mathrm{m}$ wide. " $B F \Delta R$ " values (gray bars) are local reflectivity changes measured with a $1 \mu \mathrm{m}$ beam. "DF SNR" are integrated dark-field signal to noise ratio levels scaled so that zero corresponds to the background level in the open, mask areas.

We report the peak local relative reflectivity loss (with $1-\mu \mathrm{m}$ resolution). The dark-field signal is handled differently. At each site, the signal is offset to achieve a zero average value in the area outside the repair site. The signal from an $8.1-\mu \mathrm{m}$ square area containing the repair site is then integrated to produce a response-strength value. We report this value normalized to the standard deviation of the measured background (i.e. the RMS for 8.1- $\mu \mathrm{m}$ square regions); thus the actinic dark-field value is the signal-to-noise ratio (SNR) of the measured defect site.

These two sites were selected for Fig. 4 because they exemplify two very different responses that were observed. The first site scattered light strongly, making a positive dark-field signal, combined with a severe drop in local reflectivity. Possible interpretations are that this site either strongly scattering or it redirected the reflected light into the dark-field detector signal. The second site showed the effects of strong absorption in the protection layer: the scattered light signal was lower than the surrounding area (reported as a negative value), and the reflectivity was also lower. Similar behavior is observed in the detection of surface contamination discussed in Section 3.4. 
These two sites illustrate the significance of both bright-field and dark-field actinic measurements. While dark-field measurements may be highly sensitive to small defects, large shallow defects that absorb light may be very difficult to detect, even when they cause significant bright-field reflectivity changes.

\subsection{Scanning bright-field and dark-field measurements of phase and amplitude defects}

Actinic inspection provides cross-comparison and measurement validation for commercial mask inspection tools and modeling. We have previously described one such cross-comparison ${ }^{2}$, with results that led to significant new insights. Here we briefly describe that experiment and discuss the measurement of surface particle contamination.

We performed actinic scanning inspection of a mask developed by Hoya and supplied by MIRAI (Serial No. MIRAI DEF03B $)^{10}$. The region of interest is a $150 \times 500 \mu \mathrm{m}$ programmed defect field, containing an array of buried, substrate defects, arranged into columns by size and surrounded by a border comprised of larger defects. The defects were created from 7-nm-thick CrN pads patterned onto the substrate prior to multilayer coating. Following coating, AFM-measured surface profiles ranged from 70 to $420 \mathrm{~nm}$ wide and from 3.5 to $7 \mathrm{~nm}$ high. In the region of interest, there is no absorber pattern on the mask.

Figure 5 shows scanned images of the defect array measured in bright-field and dark-field, collected simultaneously. A $2.5-\mu \mathrm{m}$ beam diameter was used in this scan. In the array there are nine columns total, with the largest defects on the left side. The defects in the right-most row suffered from resist collapse resulting in irregular shapes that appear larger than those in column eight. The figure caption explains the various scaling ranges used to reveal specific features of the data.

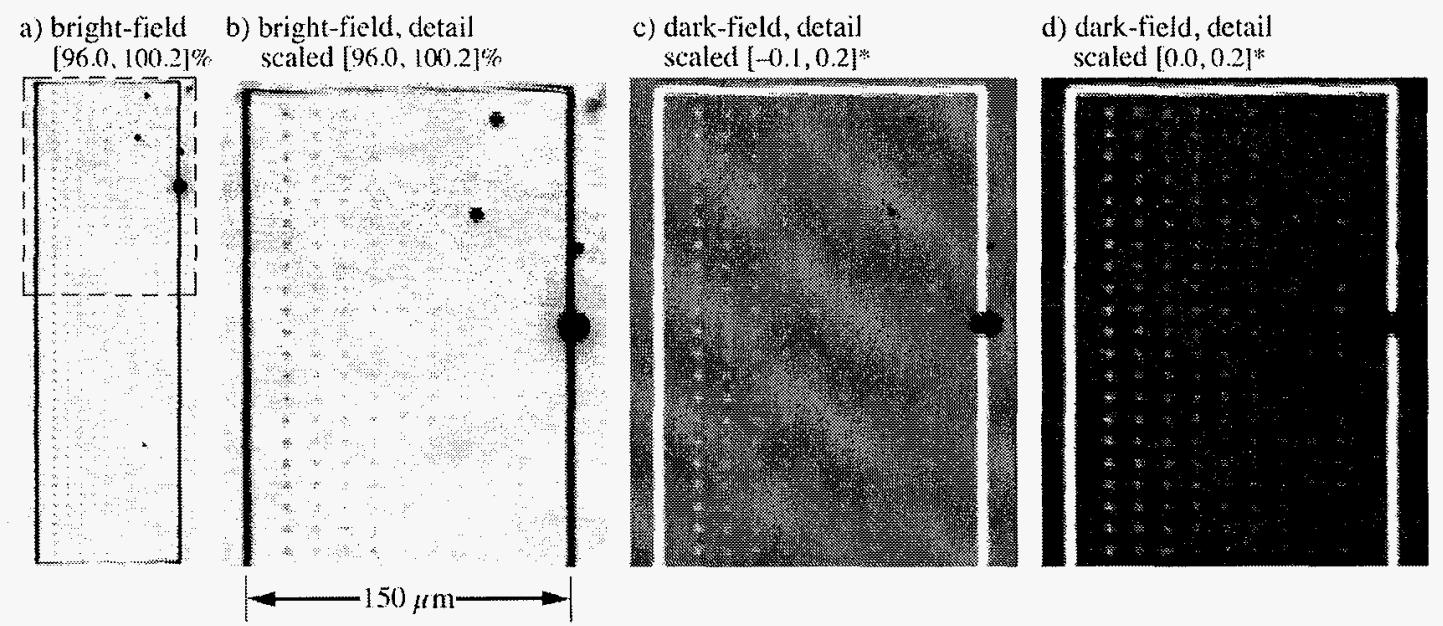

Fig. 5. Bright-field and dark-field scanned images of a programmed defect array. (a,b) are bright field scans, with (a) showing the entire region, and (b) a detail of the top portion. With the background reflectivity normalized to $100 \%$, the grayscale used for the bright-field images includes the range from $96 \%$ to $100.2 \%$ relative reflectivity. (c,d) are dark-field scans collected simultaneously with the bright-field scan. The data are scaled and normalized so that the average background level is set to 0.0 and the peak measured dark-field signal is 1.0 . (c) shows that the absorbing surface defects reduce the background scattering amplitude significantly.

An accidental contamination event prior to actinic inspection at the ALS added several particulate defects to the region of interest: these are easily seen in the upper-right sides of Figs. 5a and b, where the bright-field reflectivity shows a significant drop at the defect locations. The measurements show a $90 \%$ reflectivity drop within the largest defect, and approximately $50 \%$ decrease in the other three, nearby defects. For the buried phase defects in the array, the observed bright-field reflectivity changes were less than $2 \%$, and were typically much smaller. However, the peak magnitude of the reflectivity change is difficult to characterize because it depends sensitively on the beam footprint relative to the sizes of the defects.

The juxtaposition of phase and amplitude defects helped us to recognize the importance of the dark-field background signal for detecting absorbing surface defects that do not scatter efficiently. In uniformly bright portions of the mask, scattering from small-scale mask structure leads to a consistent yet low, noisy background signal level. This "back- 
ground' signal is scaled as gray in Fig. 5c. The dark-field signal level typically increases when the beam encounters phase defects (bright areas in Figs. $5 \mathrm{c}$ and d). However, the 1-10- $\mu \mathrm{m}$-scale particulate defects on the mask surface are accompanied by noticeable decreases in the scattered signal levels, clearly visible at the surface defect locations in Fig. $5 \mathrm{c}$ where scaled image turns black. In Fig. 5d where the dark-field image has been scaled to enhance the appearance of the buried defects and minimize the background level, the surface defects disappear into the background and are more difficult to detect.

Surface defects can thus decrease both the bright-field and the dark-field signals. If a mask were inspected using only actinic dark-field detection, then surface particles larger than several microns may be difficult to detect. To be effective actinic dark-field detection must be sensitive enough to clearly record the background scattering level with a signal-tonoise ratio high enough to observe changes associated with small, absorbing defects.

\section{SUMMARY}

It is presently unclear what role actinic mask defect inspection will play in the development and commercialization of EUV lithography. The separate requirements of mask blank inspection and patterned mask inspection place very different demands on existing and future tools. At this time, actinic inspection, conducted in the US and Japan ${ }^{11-14}$, plays an essential supporting role; this is particularly true for imaging inspection ${ }^{15}$, which has improved our understanding of defect sensitivity and printability ${ }^{1}$, and has been used to evaluate repair strategies ${ }^{16}$ among other topics.

Scanning mode inspection, as described in this article, has not been thoroughly utilized in EUVL research. Our tests on the measurements of laser-damage regions, defect repair sites, and programmed defect arrays, show the significance of both bright-field and dark-field detection methods. In the AIT, bright-field is most useful for detecting reflectivity variations on short length scales (from contamination, damage, etc.) in open field regions. Dark-field scanning is used to cross-correlate the measurements of other inspection tools, to detect phase defects, and to provide important insight for dark-field inspection system currently under development. The results of scanning tests have demonstrated that there would be significant risk associated with relying solely on bright-field or dark-field actinic inspection. The absorbing nature of micron-scale surface defects (e.g. particles and similar contamination) makes them difficult to detect in darkfield unless the tests are highly sensitive to the background reflectivity levels.

In a simple test, we investigated the at-wavelength consequences of high-resolution SEM inspection, attempting to determine if the thin layers of carbon contamination "staining" caused by SEM imaging could be detected at-wavelength. Within our measurement uncertainties, we could not detect the presence of this staining in this limited test, despite the fact that it was strongly visible in the SEM. A more thorough experiment should be designed to determine when and if SEM staining becomes detectable at-wavelength.

Intensity fluctuations during AIT scanning measurements are the primary challenge to overcome for achieving high accuracy and reducing measurement uncertainties. Scanning bright-field regions simplifies the intensity normalization that occurs in data processing and greatly improves our sensitivity to small, abrupt reflectivity variations. With sensitivity to reflectivity changes on the order of $0.1-0.2 \%$ and length scales as small as $1 \mu \mathrm{m}$, the AIT has capabilities not found in other tools, including reflectometers. Signal normalization within patterned regions is difficult in the AIT, leading to measurement uncertainties as large as several percent. Strategies to separately record illumination intensity as a normalization signal during scans are now being pursued.

\section{ACKNOWLEDGMENTS}

The authors gratefully acknowledge Yanwei Liu for his central role in the development of the actinic inspection tool, and Eric Gullikson, John Taylor, Obert Wood, Ted Liang, and Stefan Wurm for their guidance and support. Robert Gunion and Paul Denham of CXRO provided expert technical assistance with software and electronics instrumentation. The laser-damaged mask was supplied by Erdem Ultanir, formerly of Intel. The open-field mask defect repair sample was prepared by Rainer Fettig of Carl Zeiss in collaboration with Phil Seidel of SEMATECH, and we thank them for their support and many important insights. Work on the programmed defect mask was conducted in collaboration with Yoshihiro Tezuka and Tsuneo Terasawa as part of the MIRAI project; we appreciate Hoya's work in creating the mask. This work was performed under the auspices of the U.S. Department of Energy by University of California Lawrence Berkeley National Laboratory, and Lawrence Livermore National Laboratory, and was funded by SEMATECH. Research 
was conducted by scientists from the Materials Sciences Division of Lawrence Berkeley National Laboratory at the Advanced Light Source with support from the ALS Division.

\section{REFERENCES}

1. W. Cho, H.-S. Han, K. A. Goldberg, P. A. Kearney, C.-U. Jeon, "Detectability and printability of EUVL mask blank defects for the $32 \mathrm{~nm}$ HP node," Proc. SPIE 6730, 673013-1-9 (2007).

2. K. A. Goldberg, A. Barty, Y. Liu, et al., "Actinic Inspection of EUV Programmed Multilayer Defects and CrossComparison Measurements," J. Vac. Sci. \& Technol. B 24 (6), 2824-28 (2006).

3. W. Cho, P. A. Kearney, E. M. Gullikson, A. Jia, T. Tamura, A. Tajima, H. Kusunose, C.-U. Jeon, "Inspection with the Lasertec M7360 at the SEMATECH Mask Blank Development Center," Proc. SPIE 6517, 65170D (2007).

4. K. A. Goldberg, A. Barty, P. Seidel, K. Edinger, R. Fettig, P. Kearney, H. Han, O. R. Wood II , "EUV and nonEUV inspection of reticle defect repair sites," SPIE 6517, 65170C-1-7 (2007).

5. K. A. Goldberg, A. Barty, H. Han., S. Wurm, P. Kearney, P. Seidel, O. R. Wood II, B. LaFontaine, T. Liang, C. Holfeld, R. Fettig, Y. Tezuka, T. Terasawa, "Comparison of actinic and non-actinic inspection of programmed defect masks," EUVL Symposium 2006, October 18, 2006. http://goldberg.lbl.gov/presentations/p2/EUVL_2006/

6. N. Miura, A. Yamada, and M. Konagai, "Fabrication of Sub-Micron Tungsten Carbide (WCx)/Amorphous Carbon (a-C) Stacked Junction By Beam-Induced Reaction Processes," Jpn. J. Appl. Phys. 36 L, 1275-8 (1997).

7. http://henke.lbl.gov/optical_constants/

8. K. A. Goldberg, A. Barty, P. Seidel, K. Edinger, R. Fettig, P. Kearney, H. Han, O. R. Wood II , "EUV and nonEUV inspection of reticle defect repair sites," Proc. SPIE 6517, 65170C-1-7 (2007).

9. A. Barty, P. B. Mirkarimi, D. G. Stearns, D. Sweeney, H. N. Chapman, M. Clift, S. Hector, M. Yi, "EUVL mask blank repair", Proc. SPIE 4688, 385-94 (2002).

10. Y. Tezuka, M. Ito, T. Terasawa, T. Tomie, "Actinic Detection and Signal Characterization of Multilayer Defects on EUV Mask Blanks," Proc. SPIE 5567, 791-9 (2004).

11. H. Kinoshita, K. Hamamoto, N. Sakaya, M. Hosoya, T. Watanabe, "Aerial Image Mask Inspection System for Extreme Ultraviolet Lithography," Jpn. J. Appl. Phys. 46 (9B), 6113-7 (2007).

12. T. Tanaka, Y. Tezuka, and T. Terasawa, "Detection signal analysis of actinic inspection of EUV mask blanks using darkfield imaging," Proc. SPIE 6152, 61523U, (2006).

13. K. Hamamoto, Y. Tanaka, T. Yoshizumi, Y. Fukushima, H. Shiotani, N. Sakaya, M. Hosoya, T. Shoki, T. Watanabe, H. Kinoshita, "Phase defect observation using an EUV microscope," Proc. SPIE 6151, 615119 (2006).

14. Y. Tezuka, T. Tanaka, T. Terasawa, and T. Tomie, "Sensitivity-Limiting Factors of at-Wavelength Extreme Ultraviolet Lithography Mask Blank Inspection," Jpn. J. Appl. Phys. 45 (6B), 5359--72 (2006).

15. K. A. Goldberg, P. P. Naulleau, A. Barty, S. B. Rekawa, C. D. Kemp, R. F. Gunion, F. Salmassi, E. M. Gullikson, E. H. Anderson, H.-S. Han, "Performance of actinic EUVL mask imaging using a zoneplate microscope," Proc. SPIE 6730, 67305E-1-12 (2007).

16. G.-S. Yoon, H. Kim, H.-J. Oh, H.-S. Kim, H.-S. Sim, S.-H. Lee, G.-B. Kim, S.-M. Huh, K. A. Goldberg, A. Barty, S.-S. Kim, H.-K. Cho, "Evaluation of EUV Mask Repair Methods in Si-capping \& Ru-capping Blanks," EUVL Symposium 2007, Sapporo, Japan October 31, 2007. 